\title{
Pharmakologische Rückfallprophylaxe bei Alkoholabhängigkeit
}

Bernhard Croissant, Karl Mann

Zentralinstitut für Seelische Gesundheit, Klinik für Abhängiges Verhalten und Suchtmedizin, Zentralinstitut für Seelische Gesundheit Mannheim, Universität Heidelberg

psychoneuro 2004; 30 (1): 30-36

Die medikamentöse Rückfallprophylaxe der Alkoholabhängigkeit hat mit detaillierterem Verständnis der neurobiologischen Grundlagen zunehmend an Bedeutung gewonnen. So genannte Anticraving Substanzen steigern in Kombination mit psychotherapeutischen/psychosozialen Maßnahmen die bislang erreichten Behandlungserfolge beträchtlich. Besonders sind der NMDA-Rezeptormodulator Acamprosat und der Opiat-Rezeptor Antagonist Naltrexon zu nennen, deren Effekte in der Rückfallprophylaxe in zahlreichen Studien nachgewiesen werden konnten. Für Naltrexon fehlt allerdings in Deutschland die Zulassung in dieser Indikation. Werden die zur Abstinenz motivierten Patienten nach Entgiftung ein Jahr mit Acamprosat rückfallprophylaktisch behandelt, kommt es zu einer Verdoppelung der Abstinenzrate, die die eigentliche Behandlungszeit überdauert. In aktuellen Studien werden besonders geeignete Untergruppen von Patienten (,responder“) identifiziert, um dann gezielt behandelt in Zukunft den Erfolg der medikamentösen Rückfallprophylaxe weiter zu steigern.

$\mathrm{D}$ ie Alkoholabhängigkeit verläuft wie andere Suchterkrankungen chronisch rezidivierend. Ziel der Behandlung von Alkoholabhängigen ist die Motivation und Befähigung zu einer dauerhaften Abstinenz, aber auch eine Verlängerung von Abstinenzphasen bzw. eine Reduktion der Rückfallhäufigkeit kann als Behandlungserfolg gewertet werden. Bei Patienten mit „schädlichem Alkoholgebrauch" (früher „Abusus“) kann auch ein verminderter Konsum Ziel der Behandlung sein (harm reduction). Die große Heterogenität innerhalb der Gruppe der Alkoholabhängigen und die multifaktorielle Genese erfordern eine indivi- duelle Planung der Behandlung. Die akute Behandlungsphase mit der körperlichen Entgiftung im ambulanten oder stationären Rahmen zeigt sehr gute Behandlungserfolge, wenn sie in ein Konzept mit psychotherapeutischen Elementen, Aufklärung und Motivation wie bei dem Verfahren des qualifizierten Entzugs eingebunden ist. Die anschließende Entwöhnungs- und Rehabilitationsphase soll die Abstinenz längerfristig sichern. Diese Entwöhnungsbehandlung kann, je nach Schweregrad, entweder im Rahmen einer mehrmonatigen stationären oder auch ambulanten Behandlung durchgeführt werden und sieht hauptsächlich psy-

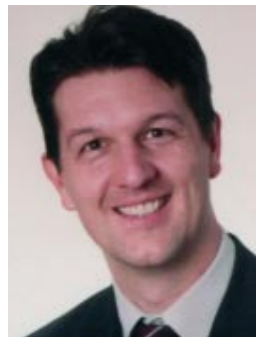

Bernhard Croissant chotherapeutische und psychosoziale Maßnahmen sowie die Anbindung an Selbsthilfegruppen vor. Der Behandlungserfolg ist bei den stationären und bei den strukturierten ambulanten Entwöhnungsbehandlungen wiederholt überprüft und nachgewiesen worden. Trotz dieser intensiven Interventionen kommt es bei ca. $40-60 \%$ der Patienten innerhalb von ein bis zwei Jahren zu einem Rückfall. Zudem wird überhaupt nur ca. $1 \%$ aller Alkoholabhängigen einer stationären Entwöhnungsbehandlung zugeführt. Der Prozentsatz ambulanter Entwöhnungsbehandlung ist noch weitaus geringer. Dies verdeutlicht die Bedeutung von zusätzlichen Behandlungsmöglichkeiten in der Postentzugsphase und hier insbesondere in der frühen Abstinenz bis zwölf Monate nach Entgiftung.

\section{Alkohol-Craving}

Dem Alkohol-Craving wird bei einem Rückfall im Rahmen einer Alkoholabhängigkeit eine Schlüsselrolle zugeschrieben. Dabei ist unter dem Begriff Craving nicht nur der subjektiv wahrgenommene Wunsch oder Druck zum Alkohol- 
konsum zu verstehen. Der Begriff Alkohol-Craving steht auch für neurobiologische/neurochemische Hirnveränderungen nach langfristigem Alkoholkonsum, die zu messbaren physiologischen Veränderungen führen und auch über unbewusste Prozesse, eventuell ohne subjektiv registrierten „Suchtdruck“, einen Rückfall verursachen können. Dabei unterscheidet man das Modell des Reward Cravings vom Modell des Withdrawal Relief Cravings (17). Unter Reward Craving versteht man durch alkoholassoziierte positive Zustände induziertes Alkoholverlangen (Belohnungstrinken). Diese opioiderg und dopaminerg vermittelten Effekte gehen mit einer Aktivierung des mesolimbischen Belohnungssystems einher, getrunken wird in Erwartung der erwünschten angenehmen Wirkungen des Alkohols (z.B. Entspannung, Wohlbefinden). Das Withdrawal Relief Craving bezeichnet Alkoholverlangen durch alkohol-assoziierte negative $\mathrm{Zu}$ stände, wie z.B. ein erwartetes Entzugssyndrom. Diese gabaerg/glutamaterg vermittelten Effekte gehen mit einer neuronalen Hyperexzitabilität einher. Getrunken wird, um diese unangenehmen Effekte (z.B. Unruhe, Zittern, Übelkeit) zu vermeiden oder $z u$ reduzieren.

\section{Medikamentöse Therapie}

Anticraving Substanzen werden seit mehreren Jahren eingesetzt, um die Abstinenzfähigkeit und damit die Prognose bei Alkoholabhängigkeit zu verbessern. Sie sollen den Alkoholrückfall verhindern, ohne selbst ein Abhängigkeitspotenzial innezuhaben oder anderweitig psychotrop zu wirken. In Deutschland sind gegenwärtig zwei Substanzen zur Behandlung der Alkoholabhängigkeit zugelassen. Neben dem aversiv wirksamen Disulfiram (z.B. Antabus ${ }^{\circledR}$ ) stehen inzwischen Substanzen zur Verfügung, die Rückfälle reduzieren. In Deutschland zugelassen in dieser Indikation ist das Acamprosat (z.B. Campral $\left.{ }^{\circledR}\right)$, in anderen EU-Staaten und den USA ist daneben auch Naltrexon (Nemexin ${ }^{\circledR}$ ) in dieser Indikation zugelassen.

\section{Abb. 1 Anteil der rückfälligen Patienten unter Naltrexon gegen Plazebo (internationale Studien), modifiziert nach Smolka}

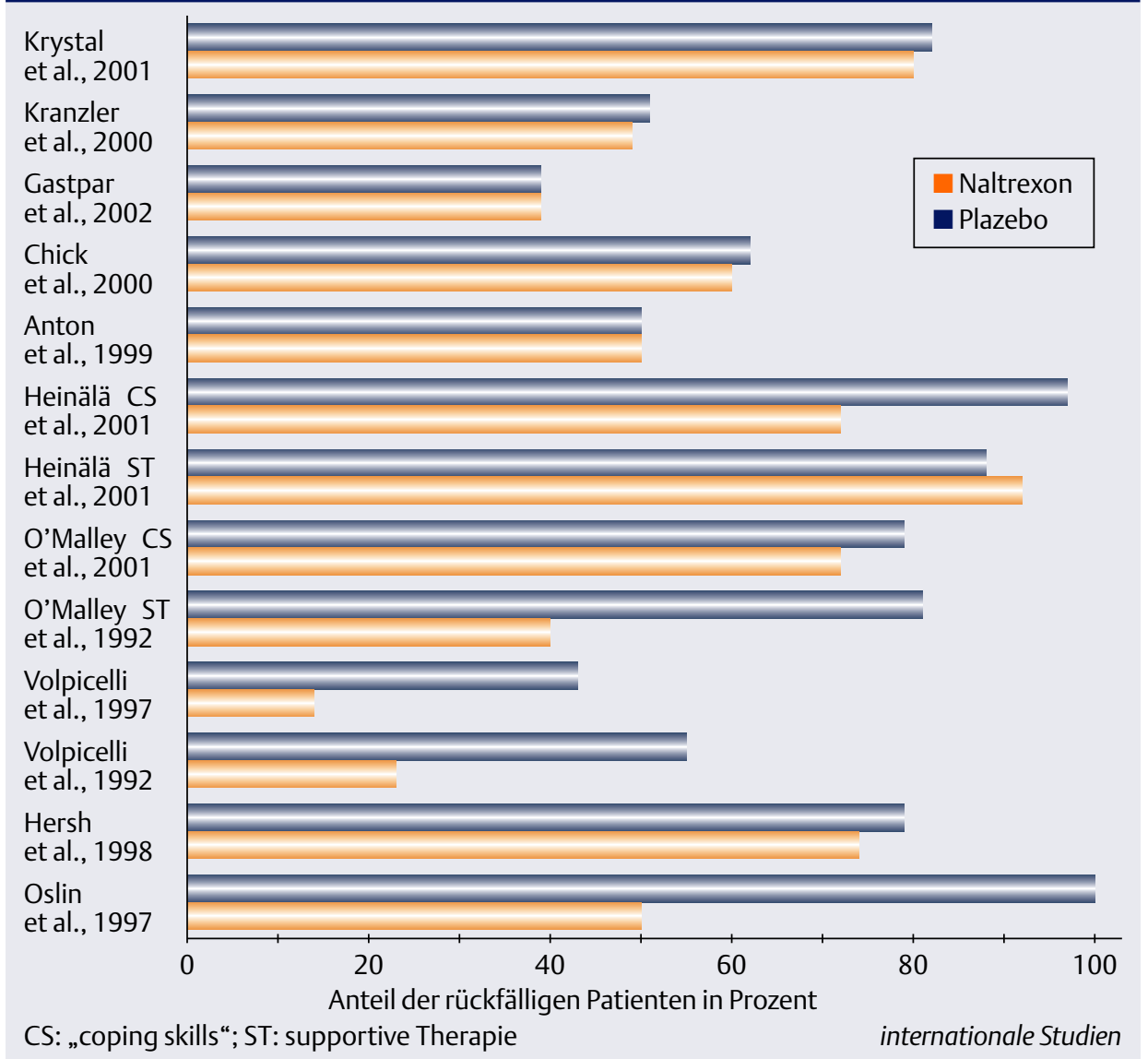

\section{Disulfiram}

Als erste medikamentöse Behandlungsmöglichkeit zur Unterstützung der Alkoholabstinenz stand das Disulfiram zur Verfügung. Diese Substanz nimmt Einfluss auf die Alkoholverstoffwechselung durch Hemmung der Aldehyd-Dehydrogenase. Nach Alkoholkonsum kommt es zu einer Akkumulation von toxischem Azetaldehyd und konsekutiv zu einer Unverträglichkeitsreaktion mit Übelkeit, Erbrechen, Kreislaufdysregulation und selten zu metabolischen Störungen wie der gefährlichen Laktazidose. Diese höchst unangenehmen Folgen sollen über ihre aversive Wirkung zur Unterdrückung des Trinkverhaltens führen. Kontrollierte klinische Studien zu der Wirksamkeit von Disulfiram konnten die Wirksamkeit nicht zweifelsfrei bestätigen $(5,7)$. Disulfiram wird deshalb nicht zur generellen Anwendung empfohlen, allerdings kann es unter strenger In- dikationsstellung, Abwägung der potenziellen Risiken und kontrollierten Rahmenbedingungen einen Platz in der medikamentösen Unterstützung der Abstinenz haben (4).

\section{Klinischer Einsatz von Disulfiram}

Nach einer Aufdosierungsphase unter absoluter Alkoholabstinenz mit 0,5-1,5 g/d an Tag 1 und 0,5-1 $\mathrm{g} / \mathrm{d}$ von Tag 2 bis Tag 10 erfolgt ab Tag 11 die Erhaltungstherapie mit 0,2-0,3 g/d (maximal 0,5 g/d). Auch die Verabreichung von $0,5 \mathrm{~g} / \mathrm{d}$ alle zwei, später alle drei Tage ist wegen der langen Halbwertszeit möglich. Die Wirkung endet im Allgemeinen ein bis vier Tage nach der letzen Einnahme, kann im Einzelfall aber bis zu 14 Tagen anhalten. Von der Durchführung eines Probetrunks ist man heutzutage weitgehend abgekommen. Die Einnahme sollte generell immer unter Sichtkontrolle durch medizinisches Personal erfolgen. 


\section{Naltrexon}

Naltrexon ist ein Opiatrezeptorantagonist von dem man annimmt, dass er die positiv verstärkenden angenehmen Effekte von Alkohol reduziert und dadurch das Craving reduziert. Durch den Wegfall der belohnenden Alkoholwirkung bzw. der Antizipation dieser Belohnung soll Naltrexon, entsprechend der o.g. Craving-Konzepte, speziell das Reward Craving reduzieren. Vor zehn Jahren zeigten zwei kleine klinische plazebokontrollierte Studien eine Reduktion der schweren Rückfälle, reduziertes Craving und weniger häufiges Trinken bei Naltrexon behandelten Patienten $(14,18)$. Diese Studien waren die Basis für die Zulassung der Substanz in den USA. Eine Abnahme in der Rate der schweren Rückfälle, gewöhnlich definiert als mehr als fünf Drinks pro Trinktag (1 Drink = 12 g Alkohol), war das konsistenteste Ergebnis, das man mit Naltrexon erzielen konnte (Abb. 1), obwohl nicht alle Studien positiv ausfielen, inklusive der größten (627 Patienten, $(10,19))$. Ein systematischer Literaturüberblick über alle publizierten Daten bis 1997 zeigt, dass Naltrexon eine konsistente Abnahme des schweren Rück- falls und der Trinkhäufigkeit der behandelten Patienten bewirkt, obwohl es anscheinend nicht die Abstinenzzeit verlängert (6).

\section{Klinischer Einsatz von Naltrexon}

Da Naltrexon in Deutschland in dieser Indikation nicht zugelassen ist, kann es nur nach Klärung der Erstattung im Rahmen der ärztlichen Behandlungsfreiheit als Heilversuch eingesetzt werden. Übelkeit, gastrointestinale Beschwerden und Kopfschmerzen stellen die häufigsten unerwünschten Arzneimittelwirkungen des insgesamt gut verträglichen Naltrexon dar. Einem eventuellen initialen Anstieg der Transaminasen folgt meistens ein noch deutlicherer Rückgang der Enzymerhöhung, was den verminderten Alkoholkonsum widerspiegelt. Eine akute Hepatitis oder schwere Leberfunktionsstörung stellen aber Kontraindikationen dar. Vor Behandlungsbeginn sollte wenigsten einige Tage Alkoholabstinenz bestehen, um das Zusammentreffen von eventuellen gastrointestinalen Nebenwirkungen und einem Entzugssyndrom zu vermeiden. Die Dosisempfehlung liegt bei konstant $50 \mathrm{mg}$ Naltrexon pro Tag. Die opiatantagonistische

\section{Abb. 2 Kontinuierliche absolute Abstinenz bis Studienende unter Acamprosat, modifiziert nach Smolka}

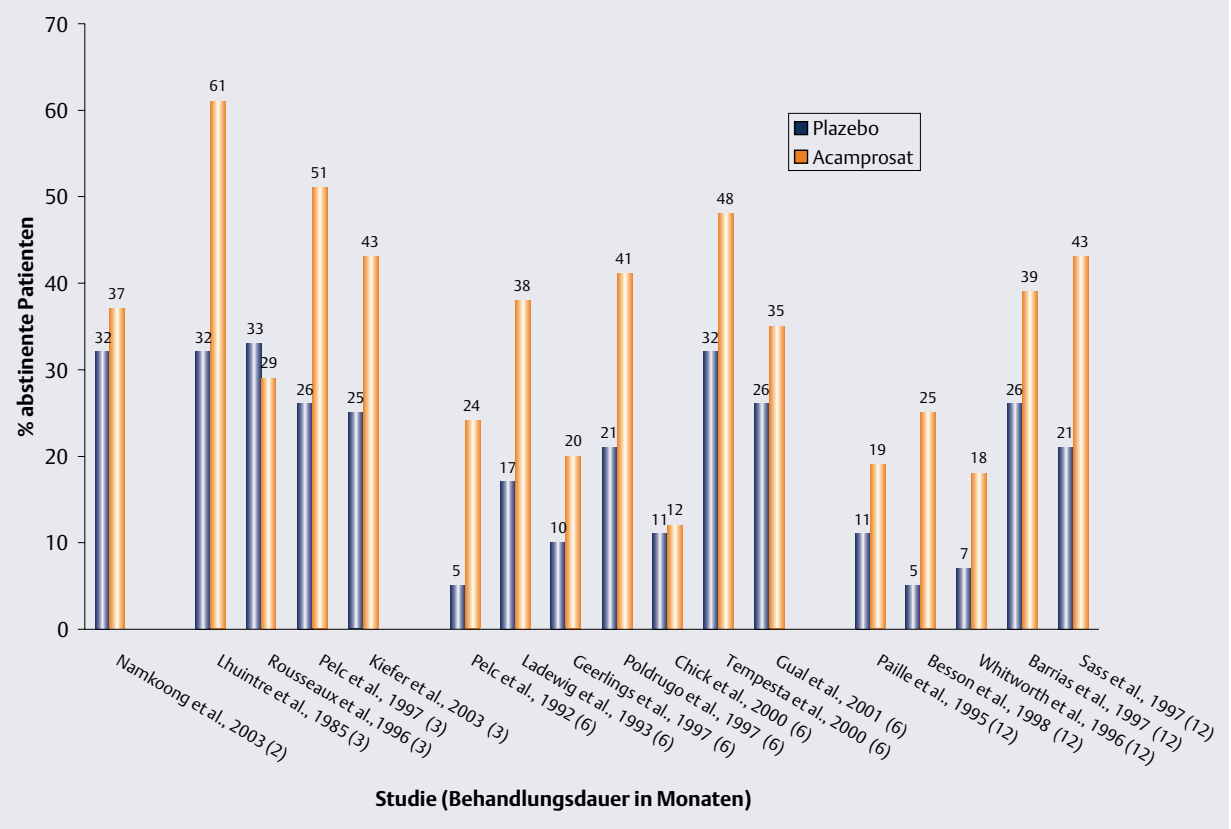


ende das Zielkriterium, für welches Acamprosat seine Überlegenheit gegenüber Plazebo zeigen konnte. Neben der Abstinenzrate war auch die Anzahl trinkfreier Tage unter Acamprosat signifikant größer als unter Plazebo. Drei Studien erbrachten allerdings keine signifikanten Resultate $(2,13,15)$. Einschränkend für die Aussagekraft dieser negativen Acamprosatstudien muss erwähnt werden, dass entweder entgegen der Verordnungsempfehlungen nicht ausschließlich abstinente Alkoholabhängige nach abgeschlossenem $\mathrm{Al}$ koholentzug, sondern auch rückfällige und trinkende Alkoholabhängige eingeschlossen worden waren (2) oder innerhalb der letzten zwei Tage vor Medikationsbeginn noch Alkohol konsumiert worden und nur über acht Wochen behandelt worden war (13). Diese negativen Ergebnisse stützen die inzwischen wissenschaftlich etablierte Auffassung, dass die Wirksamkeit von Acamprosat nicht in der Erreichung der Abstinenz, sondern im Erhalt oder Verlängerung der Abstinenz liegt und eine Behandlung über die akute poststationäre Phase hinaus fortgeführt werden sollte. An diesen Beispielen verdeutlicht sich der Einfluss der international unterschiedlichen Versorgungssysteme und Behandlungsprinzipien für die Outcome-Daten von Rückfallpräventionsstudien. Für die Beurteilung von Acamprosat im deutschen Versorgungssystem empfiehlt sich der Blick auf die Daten der methodisch anspruchsvollen deutschen Acamprosatstudie von Sass et al. (16). In dieser plazebokontrollierten Untersuchung von 272 Patienten mit einer Behandlungsdauer von 48 Wochen nach stationärem Entzug und einem Follow-up über 48 Wochen zeigte sich eine Verdoppelung der Abstinenzrate unter Acamprosat gegenüber Plazebo ( $43 \%$ vs. $21 \%$ ), die auch in der anschließenden Nachbeobachtungsphase fortbestand (39\% vs. $17 \%$ ). Darüber hinaus blieben die acamprosatbehandelten Patienten unabhängig vom Verlauf (Rückfall oder Abstinenz) signifikant länger in der Studie. Acamprosat wirkt bei der Alkoholabhängigkeit besonders dann, wenn begleitend psychotherapeutische bzw. psychosoziale Maß- nahmen stattfinden, wobei sich bislang keine der verschiedenen Methoden einer der anderen überlegen zeigen konnte (1).

\section{Klinischer Einsatz von Acamprosat}

Das seit 1989 in Frankreich und seit 1995 in Deutschland eingesetzte Acamprosat hat seine allgemein gute Verträglichkeit bereits an mehr als 1,5 Millionen Patienten bewiesen. Acamprosat führt zu keinen relevanten Interaktionen mit anderen Arzneimitteln, zu keiner erhöhten Alkoholtoxizität und besitzt kein Abhängigkeitspotenzial und keine anderen psychotropen Wirkungen. $\mathrm{Zu}$ den häufigeren Nebenwirkungen zählen Diarrhöen, andere gastrointestinale Beschwerden und Kopfschmerzen und Juckreiz. Kontraindikationen sind Schwangerschaft oder Stillzeit, Serumkreatinin > 120 $\mu \mathrm{mol} / \mathrm{l}$ bei Patienten mit Niereninsuffizienz und Vorliegen einer schweren Leberinsuffizienz. Die übliche Dosis beträgt $2 \mathrm{~g}$ täglich, aufgeteilt auf dreimal täglich zwei Tabletten á 333 mg. Für Patienten unter 60 kg Körpergewicht kann die Dosis auf $1,3 \mathrm{~g} / \mathrm{d}$ reduziert werden (empfohlen 666 - 333 - $333 \mathrm{mg}$ ), sofern unerwünschte Arzneimittelwirkungen auftreten. Therapiebeginn sollte nach Entgiftung und Motivation zur Abstinenz erfolgen. Entsprechend des in den ersten Monaten nach Entgiftung sehr hohen Rückfallrisikos empfiehlt sich eine Behandlungsdauer von zwölf Monaten. Die Behandlung sollte bei zeitlich begrenzten Rückfällen fortgeführt werden, da diese einen langfristigen Behandlungserfolg noch nicht in Zweifel stellen und aus der Kombination mit Alkohol keine Sicherheitsrisiken resultieren. Psychotherapeutische bzw. psychosoziale Maßnahmen sollten begleitend stattfinden.

\section{Therapeutische Ansätze im experimentellen Stadium}

Neben den oben genannten Möglichkeiten der medikamentösen Rückfallprophylaxe bei Alkoholabhängigkeit gibt es einige andere potenzielle Substanzen, deren Wirksamkeit bislang noch nicht ausreichend nachgewiesen werden konnte. Nalmefene ist ein Opiatrezeptor-Antagonist, ähnlich dem Naltrexon, der in einzelnen kleineren Studien gegenüber Plazebo signifikant die Rate schwerer Rückfälle reduzieren konnte (11). Das dopaminerg wirksame Neuroleptikum Tiaprid, welches in der akuten Alkoholgiftung bereits eingesetzt wird, zeigte in kleineren Studien uneinheitliche Ergebnisse, eine noch unpublizierte große Studie erbrachte keinen Vorteil gegenüber Plazebo. Das Neuroleptikum Flupentixol als Depotgabe erbrachte gar eine signifikant geringere Abstinenzrate gegenüber Plazebo, weswegen Neuroleptika in der Rückfallprophylaxe der Alkoholabhängigkeit nicht empfohlen werden können. Selektive Serotonin-Wiederaufnahmehemmer (SSRI) wurden auch auf ihre rückfallprophylaktische Wirkung bei Alkoholabhängigkeit hin untersucht. Für Fluoxetin konnte neben einigen Studien, die keinen Effekt zeigten, in einzelnen Untersuchungen eine höhere Abstinenzrate oder zumindest eine Reduktion des subjektiv erlebten Alkohol-Craving gegenüber Plazebo nachgewiesen werden. In einer Studie zum Fluoxetineffekt bei Alkoholabhängigkeit und komorbider aktueller depressiver Episode zeigte sich eine signifikante Abnahme des Alkoholkonsums, die aber wahrscheinlich als sekundärer Effekt über die Behandlung der Depression interpretiert werden muss (3). Auch für die Substanzen Citalopram und Sertralin existiert bislang noch keine eindeutige Datenlage. Odansetron, ein 5- $\mathrm{HT}_{3}$-Rezeptorantagonist, konnte einen Effekt auf Trinkmenge und Häufigkeit zeigen (8). Carbamazepin als antimanisch wirksame Substanz zeigte in einer Untersuchung mit kleiner Probandenzahl eine Reduktion des Alkoholkonsums (12), der Carbamazepineffekt in dieser Indikation bedarf allerdings noch der Bestätigung über größer angelegte Studien. Für Lithium konnte kein signifikanter Effekt in der Rückfallprophylaxe der Alkoholabhängigkeit bestätigt werden, die Lithium-spezifischen unerwünschten Arzneimittelwirkungen und Interaktionen mit anderen Substanzen sprechen zu- 
dem generell gegen den ambulanten Einsatz bei Alkoholabhängigkeit. Die Kombination von verschiedenen Substanzen stellt eine weitere potenzielle Möglichkeit zur Verbesserung der bislang unter Monotherapie erzielten Resultate dar. Für die Kombination von Naltrexon mit Acamprosat konnte eine signifikante weitere Steigerung der Abstinenzrate gegenüber den Einzelsubstanzen nachgewiesen werden (9).

\section{Schlussfolgerungen}

Die aktuelle Datenlage empfiehlt den Einsatz einer medikamentösen Rückfallprophylaxe bei Alkoholabhängigkeit. Nach der Entgiftung der alkoholabhängigen Patienten sollte bei Motivation zu vollständiger Alkoholabstinenz eine medikamentöse Therapie erwogen werden, durch welche die nichtpharmakologischen Maßnahmen der Alkoholentwöhnung unterstützt werden können. Die medikamentöse Rückfallprophylaxe sollte in ein suchtmedizinisches Behandlungskonzept eingebettet sein, welches auch weitere psychotherapeutische/psychosoziale Maßnahmen der Entwöhnungsbehandlung und Abstinenzaufrechterhaltung beinhaltet. Die Behandlungsdauer sollte zumindest den Zeitraum der statistisch größten Rückfallgefährdung abdecken und wird derzeit auf zwölf Monate angesetzt. Für einige Substanzen gibt es bislang nur erste Hinweise auf einen therapeutischen Effekt, für Naltrexon und Acamprosat ist er dagegen besonders evident, Naltrexon fehlt allerdings in Deutschland die Zulassung in dieser Indikation. Beide Substanzen werden weiterhin untersucht, u.a. in Hinblick auf die Frage, bei welchem Patienten welches Präparat besonders gut wirkt. Sollten sich in derzeit laufenden Multicenterstudien die Modellvorstellungen bestätigen, nach welchen Naltrexon besonders das RewardCraving reduziert und Acamprosat besonders auf das Withdrawal Relief Craving Einfluss nimmt, könnte nach entsprechender Identifizierung des jeweils vorherrschenden Cravingtyps die Effektivität der rückfallprophylaktischen Medikation weiter verbessert werden.
Pharmacological Relapse Prevention in Alcohol Dependence Better understanding of the neurobiological substrates of alcohol dependence has been accompanied with an increasing role of pharmacological relapse prevention. In combination with psychosocial treatment substances which reduce alcohol craving improve maintenance of $a b$ stinence. Especially the NMDA receptor modulator acamprosate and the opiate receptor antagonist naltrexone showed an effect in numerous trials. In Germany, however, naltrexone has not been approved in this indication up to now. The administration of the medication starts immediately after detoxification and should last for 12 months. The medical treatment, however, is not a replacement for a psychosocial treatment of the alcohol dependence. Compared with placebo treatment, approximately twice as many patients remain abstinent under acamprosate one year after the end of the treatment period. Current studies try to identify particular subgroups of patients responding to pharmacological relapse prevention. A specific application of these substances will optimise the outcome of rehabilitation programmes for alcohol dependent patients.

\section{Keywords:}

alcohol dependence - relapse prevention - craving - naltrexone - acamprosate

\section{Literatur}

1. Ansoms C, Deckers F, Lehert P, Pelc I, Potgieter $A$. An open study with acamprosate in Belgium and Luxemburg: results on sociodemographics, supportive treatment and outcome. Eur Addict Res 2000; 6: 132-140

2. Chick J, Howlett H, Morgan MY, Ritson B. United Kingdom Multicentre Acamprosate Study (UKMAS): a 6-month prospective study of acamprosate versus placebo in preventing relapse after withdrawal from alcohol. Alcohol and Alcoholism 2000; 35: 176-187

3. Cornelius JR, Salloum IM, Ehler JG et al. Fluoxetine in depressed alcoholics: a double-blind, placebo-controlled trial. Arch Gen Psychiatry 1997; 54: 700-705

4. Ehrenreich $\mathrm{H}$ et al. In: Mann KF (Hrsg.) Neue Therapieansätze bei Alkoholproblemen. Lengerich, Pabst, 2002: 107-118

5. Fuller RK et al. Disulfiram treatment of alcoholism. A Veterans Administration coo- perative study. JAMA 1986; 256: 1449-1455 6. Garbutt JC, West SL, Carey TS et al. Pharmacological treatment of alcohol dependence: a review of the evidence. JAMA 1999; 281: 1318-1325

7. Johnsen J, Morland J. Disulfiram implant: a double-blind placebo controlled follow-up on treatment outcome. Alcohol Clin Exp Res 1991; 15: 532-536

8. Johnson BA, Roace JD, Javors MA et al. Ondansetron for reduction of drinking among biologically predisposed alcoholic patients: a randomised clinical trial. JAMA 2000; 284: 1016-1017

9. Kiefer $\mathrm{F}$ et al. Comparing and combining naltrexone and acamprosate in relapse prevention of alcoholism: a double-blind, placebo-controlled study. Arch Gen Psychiatry 2003; 60: 92-99

10. Krystal JH, Cramer JA, Krol WF et al. Naltrexone in the treatment of alcohol dependence. N Engl J Med 2001; 345: 1734-1739

11. Mäkelä R, Kallio A, Karhuvaara S. From the Finnish Nalmefene Study Group. Nalmefene in the treatment of heavy drinkers. $4^{\text {th }}$ ISAM Abstract 2001; Trieste

12. Müller TI, Stout RL, Rudden S et al. A double-blind placebo-controlled pilot study of carbamazepine for the treatment of alcohol dependence. Alcohol Clin Exp Res 1997; 21: 86-91

13. Namkoong K, e BO, Lee PG, Choi MJ, Lee E. Acamprosate in korean alcohol-dependent patients: a multi-centre, randomized, double-blind, placebo-controlled study. Alcohol and Alcoholism 2003; 38: 135-141 14. O'Malley SS, Jaffe AJ, Chang G et al. Naltrexone and coping skills therapy for alcohol dependence: a controlled study. Arch Gen Psychiatry 1992; 49: 881-887

15. Roussaux JP, Hers D, Ferauge M. Does acamprosate diminish the appetite for alcohol in weaned alcoholics? J Pharm Belg 1996; 51: 65-68

16. Sass H, Soyka M, Mann K, Zieglgansberger W. Relapse prevention by acamprosate. Results from a placebo-controlled study on alcohol dependence. Arch Gen Psychiatry 1996; 53: 673-680

17. Verheul R, van den Brink W, Geerlings $P$. A three-pathway psychobiological modell of craving for alcohol. Alcohol and Alcoholism 1999, 34, 197-222

18. Volpicelli JR, Alterman Al, Hayashida M, O'Brien CP. Naltrexone in the treatment of alcohol dependence. Arch Gen Psychiatry 1992; 49: 876-880

19. Gastpar M et al. Lack of efficacy of naltrexone in the prevention of alcohol relapse: results from a German multicenter study. J Clin Psychopharmacol 2002; 22(6): 592-598

\section{Korrespondenzadresse}

Dr. med. Bernhard Croissant Zentralinstitut für Seelische Gesundheit Klinik für Abhängiges Verhalten und Suchtmedizin

Leitender Oberarzt der Klinik (komm.) J5

68159 Mannheim croissant@zi-mannheim.de 